Research Paper

\title{
Development of a Model for the Prediction of Treatment Response of Uterine Leiomyomas after Uterine Artery Embolization
}

\author{
Youn-Jee Chung1, So-Yeon Kang1, Ho Jong Chun², Sung Eun Rha², Hyun Hee Cho'1, Jang Heub Kim¹, \\ Mee-Ran Kim ${ }^{1}$ \\ 1. Department of Obstetrics and Gynecology, Seoul St. Mary's Hospital, College of Medicine, The Catholic University of Korea, Seoul, Korea. \\ 2. Department of Radiology, Seoul St. Mary's Hospital, College of Medicine, The Catholic University of Korea, Seoul, Korea. \\ $\square$ Corresponding author: Mee-Ran Kim, MD., Ph.D., Professor, Department of Obstetrics and Gynecology, Seoul St. Mary's Hospital, College of Medicine, The \\ Catholic University of Korea, 222 Banpo-daero, Seocho-Gu, Seoul, 06591, Republic of Korea. Tel:82-2-2258-6170; Fax: 82-2-595-1549; E-mail: mrkim@catholic.ac.kr \\ (c) Ivyspring International Publisher. This is an open access article distributed under the terms of the Creative Commons Attribution (CC BY-NC) license \\ (https://creativecommons.org/licenses/by-nc/4.0/). See http://ivyspring.com/terms for full terms and conditions.
}

Received: 2018.07.22; Accepted: 2018.09.26; Published: 2018.11.23

\begin{abstract}
Background: Uterine artery embolization (UAE) is one of the minimally-invasive alternatives to hysterectomy for treatment of uterine leiomyomas. There are various factors affecting the outcomes of UAE, but these have only been sporadically studied.

Study Objective: To identify factors associated with the efficacy of UAE for the treatment of uterine leiomyoma, and to develop a model for the prediction of treatment response of uterine leiomyomas to UAE.

Study design: A retrospective cohort study (Canadian Task Force Classification II-2)

Patients: One hundred ninety-eight patients with symptomatic uterine leiomyomas.

Intervention: UAE

Measurements and Main Results: Among 198 leiomyoma patients who were treated with UAE, 104 who underwent pelvic magnetic resonance imaging (MRI) with diffusion-weighted imaging were selected for developing prediction model. Variables that were statistically significant from the univariate analysis were: location of leiomyoma, total number of lesions, sum of leiomyomas diameters, T2 signal intensity of largest leiomyoma, and T2 leiomyoma:muscle ratio. After a logistic regression analysis, leiomyoma location and $\mathrm{T} 2$ signal intensity of the largest leiomyoma were found to be statistically significant variables. Using intramural myomas defined as controls, submucosal leiomyomas showed a greater response to UAE with an odds ratio of 7.6904. The odds ratio of T2 signal intensity with an increase in signal intensity of 10 was 1.093 . Using these two variables, we developed a prediction model. The AUC in the prediction model was 0.833 , and the AUC in the validation set was 0.791 .

Conclusion: We identified that submucosal leiomyomas and those leiomyomas that show high signal intensity on T2-weighted imaging will exhibit a greater response to UAE. Prediction models are clinically helpful in selecting UAE as an appropriate treatment option for managing uterine leiomyoma.
\end{abstract}

Key words: uterine artery embolization, leiomyoma, magnetic resonance imaging, decision modeling

\section{Introduction}

Uterine leiomyomas are the most common benign gynecologic tumors. The prevalence of uterine leiomyoma has been reported as high as $20 \%$ to $40 \%$
$[1,2]$. This benign disease tends to increase in size until menopause. Although $50 \%$ of patients are asymptomatic, uterine leiomyoma is one of the 
common causes of hysterectomy in premenopausal women [3]. With the modern increase in later marriages and pregnancies, uterus-preserving treatments have gained favor as an alternative to hysterectomy. Patients also generally choose procedures that avoid the risks of surgical complications and general anesthesia. Uterine artery embolization (UAE) is one of the new minimallyinvasive alternatives to hysterectomy, and over 22,000 cases are performed per year in the United States [4]. UAE does not cause post-procedure adhesions and can treat all leiomyoma lesions simultaneously. Leiomyomas that necrotized and complete infarcted after UAE procedure represented no recurrence during the long-term follow-up period. UAE can control heavy vaginal bleeding within 24 hours after the procedure in $90 \%$ of patients [5].

Although demand for UAE has increased, research about predictive factors of the response after UAE has been limited. There are various factors that could affect post-UAE outcomes, such as location, size, and number of leiomyomas [6-8]. Magnetic resonance imaging (MRI) is the most precise imaging method for localizing leiomyomas and for differentiating lesions from other diseases such as adenomyosis, solid ovarian tumors, and leiomyosarcomas [9]. MRI before UAE is also very helpful in predicting success and adverse effects of the procedure $[10,11]$. The degree of vascularity, contrast enhancement, signal intensity in T1- and T2-weighted imaging, and apparent diffusion coefficient (ADC) in diffusion weighted imaging were factors associated with the efficacy of UAE [12-15].

We expected that it could be possible to predict uterine leiomyoma treatment response to UAE by analyzing various factors from pre-procedure MRIs. We performed this study to design a prediction model for UAE by using select characteristics from pre-procedure MRI.

\section{Materials and Methods}

\section{Study subjects}

We analyzed medical record and MRI findings of uterine leiomyoma patients who underwent UAE for the treatment of symptomatic uterine leiomyoma from December 1, 2008 to August 31, 2014 at Seoul St. Mary's Hospital. Among 198 patients, 104 who received MRIs with diffusion-weighted imaging were selected for this study. Among the patients who underwent UAE, those with uterine adenomyosis or pelvic congestion syndrome, and those who did not perform a post-UAE follow-up MRI were excluded. Approval for the use of information from medical records and MRI findings was granted by the
Institutional Review Board of Seoul St. Mary's Hospital (KC13RASI0311).

\section{Determination of groups and variables for analysis}

Among 104 patients, 70\% of the patients were randomly assigned to a model development group (73 patients), and the remaining patients formed the model evaluation group (31 patients). The former group was used to provide data to develop a model for the prediction of treatment response of uterine leiomyomas after UAE, and the latter group was used to objectively validate the model.

We defined the high-response group to be those patients in whom the total uterine volume (TUV), including the myoma, and the largest leiomyoma volume (LMV) were both reduced below the median values after UAE. The remaining subjects were categorized as the low-response group. We analyzed characteristics of the model development group, including age, body mass index (BMI), marital status, and parity. We measured TUV, LMV, location of the largest leiomyoma, T1 and T2 signal intensity, the degree of contrast enhancement of the largest leiomyoma, b1000 and ADC values in pre-UAE MRI, and variables except b1000 and ADC values in post-UAE MRI and analyzed the differences before and after procedure.

TUV and LMV were calculated using the formula for ellipsoid volume (width $x$ length $x$ height $x \pi / 6)$. The location of the largest leiomyoma was categorized as either subserosal, intramural, or submucosal type. We also classified the largest leiomyoma according to the leiomyoma subclassification system of the International Federation of Gynecology and Obstetrics (FIGO) [16]. Signal intensities at T1, T2, and b1000 were classified as high, iso, or low signal intensity in comparison to normal myometrium. The degree of contrast enhancement of the largest leiomyoma was classified as either heterogeneous or homogenous. The largest leiomyoma's ADC and T2 signal intensities were calculated using regions of interest (ROI). To more objectively compare leiomyomas between patients, using a common denominator was necessary. Therefore, the T2 leiomyoma:muscle ratio was used to predict reduction of uterine leiomyoma size [17].

\section{Model analysis for prediction of treatment response of uterine leiomyomas after uterine artery embolization}

Through analysis of the model development group, we determined variables that will be included in the final estimation model and analyzed receiver operating characteristics (ROC) according to the final 
model. We classified post-UAE response of uterine leiomyomas into quartiles and verified the model using a box plot.

\section{Uterine artery embolization}

Interventional radiologists performed UAE in all patients in the same manner. After skin preparation of the right inguinal region, $10 \mathrm{ml}$ of $2 \%$ lidocaine $\mathrm{HCl}$ was injected locally and the skin was incised. The right femoral artery was punctured with an 18G angiocatheter needle and a $5.5 \mathrm{Fr}$ arterial sheath was then inserted. $10 \mathrm{ml}$ of saline solution mixed with heparin was then injected through the sheath. Both uterine arteries were selected using a 5 Fr RUC catheter (RUC; Cook, Bloomington, IN, USA), and following a coaxial microcatheter, pelvic angiography was performed with a $5 \mathrm{Fr}$ pigtail catheter and a 0.035-inch Terumo wire (Terumo, Tokyo, Japan). The selective uterine arteriogram was obtained and 500 700 micron polyvinyl alcohol particles (Contour; Boston Scientific, Marlborough, MA, USA) were then injected through the microcatheter for embolization. Post-embolization pelvic angiography was then performed. The endpoint of UAE was occlusion of the perifibroid plexus, but with preserved sluggish antegrade flow in the uterine arteries. Abdominal aortography was done at the end of the procedure, and if recanalization of the artery was observed, additional embolization was done until arterial flow was appropriately slowed.

\section{Statistical Analysis}

After UAE, if both TUV and LMV were reduced from the median values, then we determined these patients to be the high-response group, while the other subjects were denoted as low-response. The data are presented as mean \pm standard deviation (SD) in continuous variables or numbers and percentages in categorical variables. The subjects' characteristics were compared depending on response after UAE by use of independent-sample Student's t-tests for continuous variables and chi-square tests for categorical variables. For continuous variables that did not show a normal distribution, we used a log conversion for the analysis.

Final risk factors that were included in our estimation model were obtained from logistic regression analysis. For considering small sample size, we used Firth's Penalized Likelihood approach in SAS software [18]. After building an estimation model, we validated the model using an ROC curve and area under the curve (AUC). In this study, statistical analyses were performed by using SAS software (version 9.3; SAS Institute, Cary, NC, USA).
A $P$ value $<0.05$ was considered as statistically significant.

\section{Results}

\section{Baseline characteristics according to post-UAE response of uterine leiomyoma}

Seventy-three patients in the model development group were divided into either a high-response group, in which both TUV and LMV were reduced more than median values, or a low-response group. We analyzed variables that affected the treatment response of uterine leiomyoma after UAE. Pelvic MRIs for treatment follow-up were performed an average of 97.9 days after UAE. Median value of mean TUV volume reduction rate was $35.5 \%$ and that of mean LMV volume reduction rate was $46.1 \%$. In both low- and high-response groups, there were no significant differences with regards to rate of multiparous women, age, or BMI (Table 1).

Table 1. Demographic characteristics of study populations

\begin{tabular}{llll}
\hline & \multicolumn{2}{l}{ Uterus and myoma volume } & \\
\cline { 2 - 3 } & $\begin{array}{l}\text { Low-response group } \\
(\mathbf{n = 4 8 )}\end{array}$ & $\begin{array}{l}\text { High-response group } \\
(\mathbf{n = 2 5 )}\end{array}$ & $\boldsymbol{P}$-value \\
\hline Parous women $\mathbf{( \% )}$ & $25(52.1)$ & $13(52.0)$ & 0.995 \\
Age $(\mathbf{y r s})$ & $44.5 \pm 3.8$ & $42.6 \pm 5.9$ & 0.150 \\
$\mathbf{B M I}\left(\mathbf{k g} / \mathbf{m}^{2}\right)$ & $23.1 \pm 3.2$ & $23.3 \pm 2.9$ & 0.830 \\
\hline
\end{tabular}

We analyzed various factors associated with treatment outcomes in pelvic MRIs, which were performed before UAE (Table 2). The most common location in the high-response group $(P=0.002)$ was submucosal, seen in 13 patients $(52 \%)$. Total myoma number was fewer in the high-response group than the low-response group (4.2 vs. $6.8, P=0.009)$. The sum of leiomyomas diameters was also smaller in the high-response group than the low-response group $(14.6 \pm 10 \mathrm{~cm}$ vs. $21.4 \pm 12.4 \mathrm{~cm}, P=0.021)$. Signal intensity of the largest leiomyoma in T2-weighted imaging was higher in the high-response group than the low-response group $(409.3 \pm 223.9$ vs. $256.7 \pm$ 140.4, $P=0.004)$. T2 leiomyoma:muscle ratio, defined as between the largest leiomyoma and the gluteus muscle, was higher in the high-response group than the low-response group $(1.9 \pm 1$ vs. $1.4 \pm 0.6, P=$ 0.019 ).

Distribution of variables that were statistically significant from the univariate analysis is presented as a box plot, according to the four groups by combination of volume reduction of TUV and LMV (Figure 1). When both TUV and LMV were included in the low-response group, this defined group 1. If TUV was in the high-response group and LMV in the low-response group, then this defined group 2. If TUV was in the low-response group and LMV was in the 
high-response group, this defined group 3. When both TUV and LMV were included in the high-response group, this defined group 4 . Submucosal leiomyomas represented the highest proportion of group 4 (Figures $1 \mathrm{~A}$ and $\mathrm{B}$ ). Total number of leiomyoma lesions and the sum of leiomyomas diameters provided the least contribution to group 4 (Figures $1 \mathrm{C}$ and D). The largest leiomyoma's T2 signal intensity and T2 signal intensity ratio were highest in group 4 (Figures 1E and F).

Table 2. Clinical characteristics of study populations based on the combination of uterine volume change and leiomyoma volume change

\begin{tabular}{|c|c|c|c|}
\hline & \multicolumn{2}{|c|}{ Uterus and myoma volume } & \multirow[b]{2}{*}{$\begin{array}{l}P- \\
\text { value }\end{array}$} \\
\hline & $\begin{array}{l}\text { Low-response } \\
\text { group } \\
(\mathrm{n}=48)\end{array}$ & $\begin{array}{l}\text { High-response } \\
\text { group } \\
(\mathrm{n}=25)\end{array}$ & \\
\hline Location of myoma (\%) & & & 0.002 \\
\hline Subserosal & $4(8.3)$ & $4(16)$ & \\
\hline Intramural & $35(72.9)$ & $8(32)$ & \\
\hline Submucosal & $9(18.8)$ & $13(52)$ & \\
\hline FIGO classification $(\%)$ & & & 0.006 \\
\hline 6 & $4(8.3)$ & $4(16)$ & \\
\hline $4,5,2-5$ & $34(70.8)$ & $8(32)$ & \\
\hline $0,1,2$ & $10(20.8)$ & $13(52)$ & \\
\hline Uterine volume $\left(\mathrm{cm}^{3}\right)$ & $479.6 \pm 204.5$ & $422.4 \pm 178.9$ & 0.242 \\
\hline Total myoma number* & $6.8(5.6,8.2)$ & $4.2(3.1,5.8)$ & 0.009 \\
\hline $\begin{array}{l}\text { Sum of diameters of myoma } \\
\text { (cm) }\end{array}$ & $21.4 \pm 12.4$ & $14.6 \pm 10$ & 0.021 \\
\hline Diameter of largest myoma (cm) & $6.8 \pm 1.8$ & $6.8 \pm 2.7$ & 0.907 \\
\hline Largest myoma volume* $(\mathrm{cm} 3)$ & $\begin{array}{l}100.2 \\
(75.6,132.7)\end{array}$ & $88(60,129.1)$ & 0.584 \\
\hline \multicolumn{4}{|l|}{ Largest myoma T2 SI (\%) } \\
\hline homogeneous & $18(37.5)$ & $10(40)$ & 0.835 \\
\hline heterogeneous & $30(62.5)$ & $15(60)$ & 0.835 \\
\hline Largest myoma T2 SI & $256.7 \pm 140.4$ & $409.3 \pm 223.9$ & 0.004 \\
\hline T2 SI myoma-muscle-ratio & $1.4 \pm 0.6$ & $1.9 \pm 1$ & 0.019 \\
\hline b1000 (\%) & & & 0.019 \\
\hline low & $31(64.6)$ & $9(36)$ & \\
\hline iso & $13(27.1)$ & $8(32)$ & \\
\hline high & $4(8.3)$ & $8(32)$ & \\
\hline $\operatorname{ADC}\left(\mathrm{mm}^{2} / \mathrm{s}\right)$ & $1180.7 \pm 207.5$ & $1274.7 \pm 224.8$ & 0.079 \\
\hline
\end{tabular}

\section{Development of the model for prediction of post-UAE response of uterine leiomyomas}

To help predict treatment response of uterine leiomyoma after UAE, a logistic regression analysis was performed for selecting the final variables that would be included in the estimation model (Table 3). Variables that were statistically significant from the univariate analysis were location of leiomyoma, total number of leiomyomas, sum of leiomyomas diameters, T2 signal intensity of largest leiomyoma, and T2 leiomyoma:muscle ratio. After logistic regression analysis, leiomyoma location and T2 signal intensity of the largest leiomyoma were found to be the significant variables. If intramural leiomyomas were denoted as the control group, then submucosal leiomyomas exhibited a greater effect after UAE with an odds ratio of 7.6904. The odds ratio of T2 signal intensity for every increase of 10 in signal intensity was 1.093. Using these two variables, we could develop a prediction model with the equation shown below.

$$
\begin{gathered}
y=e^{\left(-2.9842+0.4548 \times x_{1}+0.7928 \times x_{2}+0.0885 \times x_{3}\right)} \\
\text { Subserosal leiomyoma: } x_{1}=1, x_{2}=0 \\
\text { Intramural leiomyoma: } x_{1}=-1, x_{2}=-1 \\
\text { Submucosal leiomyoma: } x_{1}=0, x_{2}=1 \\
x_{3}=\frac{\text { Largest leiomyoma T2 SI }}{10} \\
e=2.718281828
\end{gathered}
$$

To verify the validity of this model, an ROC curve and AUC was used. The AUC in the prediction model was 0.833 , and 0.791 in the validation set. This data meant that this model could accurately predict treatment response of UAE $83.3 \%$ of the time. By using the validation set, the model was shown to predict treatment response of UAE with $79.1 \%$ accuracy (Figure 2A). To estimate the probability distribution of the prediction model, a histogram was used. We substituted the validation set in the equation to calculate the probability of the prediction model. To construct a histogram, we divided the calculated probability into quartiles. A value of $\mathrm{y}$, the calculated probability, less than $7.5 \%$, defined Q1. If the value of $y$ was $7.5-14.7 \%$, this defined Q2. If the value of $y$ was $14.7-53.4 \%$, this defined Q3. A value of y over $53.4 \%$ defined Q4. If the value of $\mathrm{y}$ was in Q1, the prediction value was $0 \%$. A value of $y$ in Q2 correlated with a prediction value of $25 \%$. If the value of $y$ belonged to Q3, the prediction value was $37.5 \%$. If the value of $y$ was in $Q 4$, the prediction value was $62.5 \%$. For example, if the location and the T2 signal intensity of the largest leiomyoma were substituted in the equation, and the calculated y value was $3 \%$, then this would result in a Q1 value. Further, if the prediction value was $0 \%$, then a patient could not expect the positive effects of UAE. If the calculated y value was $55 \%$, it belongs to Q4. This would correlate with a patient having a $62.5 \%$ probability of belonging to the high response group (Figure $2 \mathrm{~B}$ ).

Table 3. Logistic regression analyses about predictors of UAE response

\begin{tabular}{lllll}
\hline & OR & 95\% CI & P-value \\
\hline Location of myoma & & & & 0.010 \\
Subserosal & 5.487 & 0.794 & 37.392 & \\
Intramural & 1 & & & \\
Submucosal & 7.694 & 1.994 & 29.682 & \\
Largest myoma T2 SI & 1.093 & 1.037 & 1.151 & 0.001 \\
\hline
\end{tabular}


A

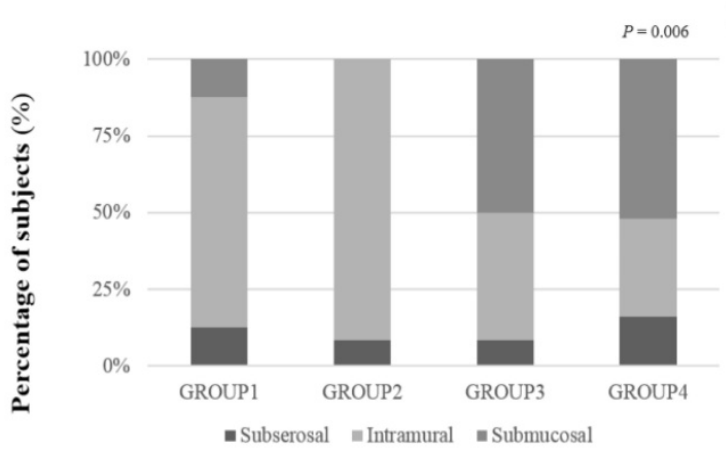

C

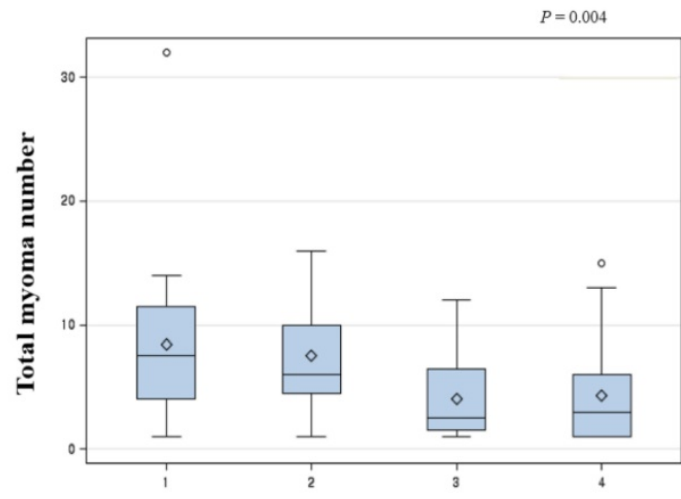

E

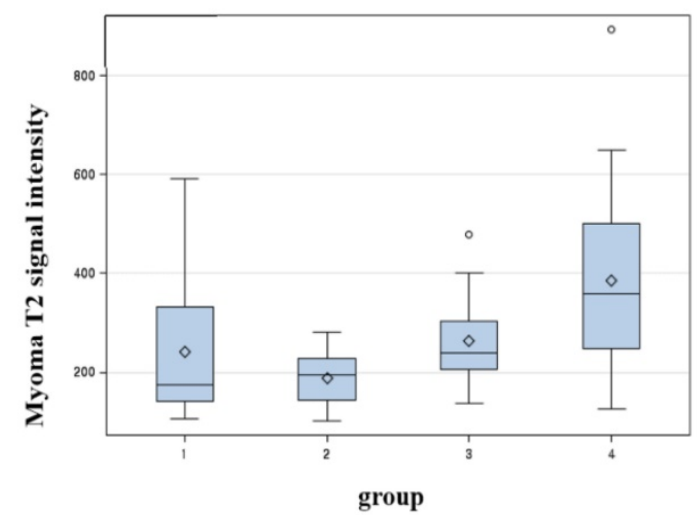

B

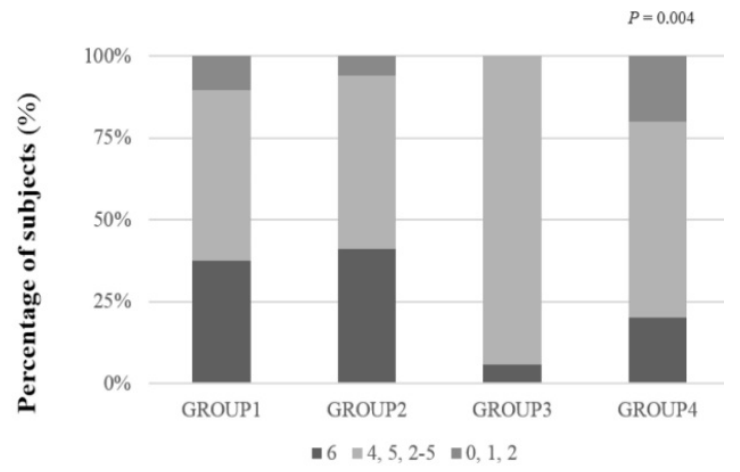

FIGO classification

D

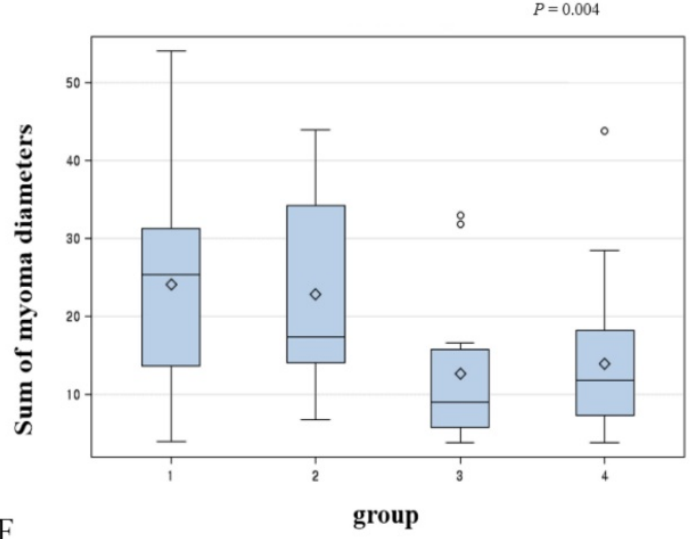

F

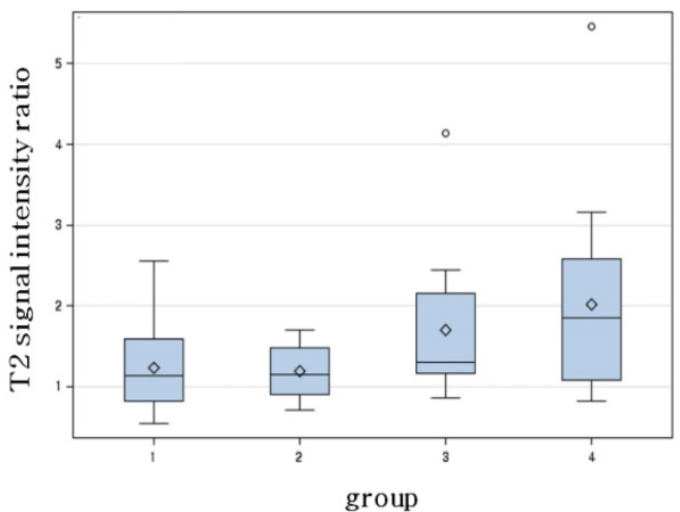

Figure 1. The distribution of the variables according to the four groups. A. location of leiomyoma, B. location of leiomyoma by FIGO classification, C. total leiomyoma number, D. sum of leiomyomas diameters, E. leiomyoma T2 signal intensity, F. T2 signal intensity ratio (group 1: both TUV and LMV are in low-response group, group 2: TUV in high-response group, TMV in low-response group, group 3: TUV in low-response group, TMV in high-response group, group 4: both TUV and LMV are in high-response group)

\section{Discussion}

UAE is a minimally invasive procedure for alleviating symptoms associated with and treating uterine leiomyoma. It is also a very effective uterinepreserving treatment option for uterine leiomyoma patients who wish to preserve their uterus [19].

Studies about factors associated with the effect of UAE have thus been limited. According to location, submucosal leiomyomas displayed the most significant volume reduction after UAE [20]. However, other studies have shown that leiomyoma location does not have significant clinical impact on UAE outcome [21, 22]. Size of uterine leiomyoma is not a limiting factor in UAE [23-25]. Another study showed that hypervascular and single leiomyomas experienced better UAE outcomes than hypovascular or multiple leiomyomas [26]. If a leiomyoma was over $90 \%$ infarcted after UAE, the patient showed better symptom control than those with a lower infarction rate [27]. Factors associated with the effects of UAE have only been sporadically studied, and thus there are no systematic prediction models that consider all the factors. 
A

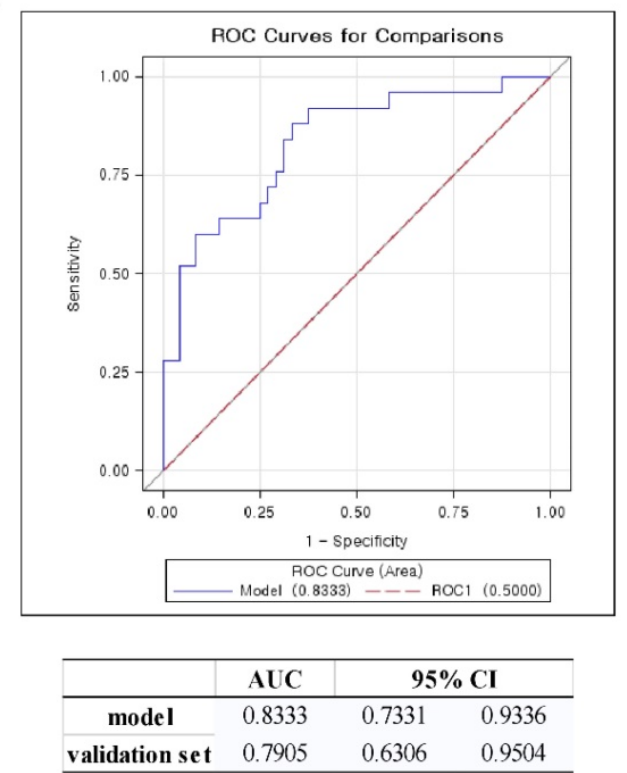

B

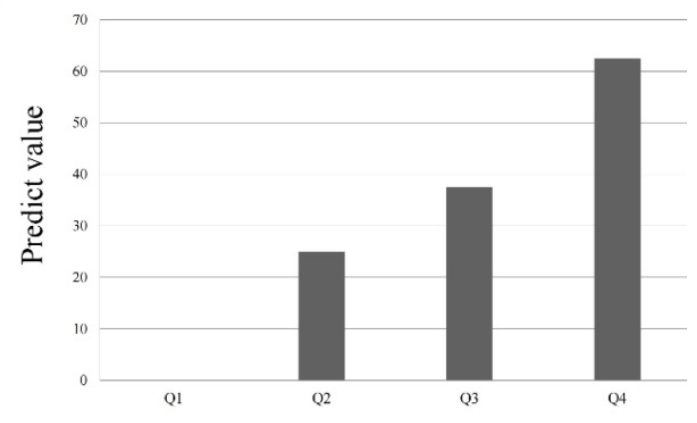

Figure 2. Validation of the model using ROC curve and box plot. A. ROC curve for validation of the model, B. prediction value for validation of the model (Q1<7.4\%, Q2=7.4-14.7\%, Q3=14.7-53.4\%, Q4>53.4\%)

In this study, we analyzed various factors obtained from pre-procedure MRIs to predict treatment response of uterine leiomyoma after UAE. As a result, we found that submucosal leiomyomas showed a better response to UAE, and if total leiomyoma number and the sum of leiomyomas diameters were smaller, the response after UAE was also improved.

The results of the present study correspond well with those of an earlier study that reported that submucosal leiomyomas respond better to UAE. This phenomenon was explained by the distribution of the vasculature in the myometrium. The radial artery, the main branches of intramural uterine artery, supplied much more blood flow to the central two-thirds of the myometrium, including the endometrium. Therefore, UAE is more effective in the treatment of submucosal leiomyomas than intramural or subserosal leiomyomas [28]. A large uterine volume before UAE is known to be a poor prognostic factor. This is related with the fact that a large uterine volume before UAE means that embolic particles need to be delivered to larger regions [10]. This finding was similar to our study results that a smaller number of total leiomyomas and smaller sum of leiomyomas diameters led to a greater response to UAE.

Signal intensities of pelvic MRIs before UAE also correlated with treatment response of leiomyoma after UAE. Treatment response after UAE was much improved when the uterine leiomyoma showed a high signal intensity on T2-weighted imaging and the ratio of T2 signal intensity between uterine leiomyoma and gluteus muscle was high. Leiomyomas that showed a high signal intensity on T2-weighted imaging and which showed greater treatment response after UAE had a close correlation with the cellularity and vascularization of the lesions [29]. Cellular leiomyomas that showed high signal intensity on T2-weighted imaging and this histologic type displayed a greater response to UAE [30]. These results are consistent with the present study. One study showed that the ratio between the uterine leiomyoma and skeletal muscle was higher when the treatment response was greater [17].

We developed a model for predicting treatment response of uterine leiomyoma after UAE. As a result, we could design a model using the location and T2 signal intensity of leiomyoma. In general, if the AUC in a ROC curve is above 0.7 , it is accepted as appropriate in an epidemiologic study. The prediction model that resulted from this study had an AUC of 0.833 , and examination using validation set showed the AUC to be 0.791 . It showed that this model is accepted as appropriate. A previous study about the prediction of early response to UAE in leiomyomas using $\mathrm{T} 1$ signal intensity and degree of contrast enhancement had AUC values of 0.712 for $\mathrm{T} 1$ signal intensity and 0.731 for degree of contrast enhancement [28]. Compared to this study, our study found that T2 signal intensity and lesion location were more appropriate for prediction of treatment outcome.

This study has several limitations. First, this study has a small sample size. Because of its small sample size, there were difficulties in producing a prediction model effectively. For overcoming this limitation, we used odds ratios from Firth's Penalized 
Likelihood method [18]. This method is suitable for medical studies especially for small sample size. Second, this is a retrospective study. Third, there was a lack of specific information about symptom relief after UAE. If study subjects had been increasing continuously, and long-term follow-up study was adequately performed, further studies about the factors associated with treatment response after UAE could be possible. The effect of associated factors on the recurrence or continuation of the therapeutic effect could then be evaluated in the future.

The results of this study suggest that submucosal leiomyomas and leiomyomas that show high signal intensity on T2-weighted imaging will experience a greater response after UAE. Employing a prediction model in clinical practice would be incredibly useful in selecting UAE as an appropriate treatment option for managing uterine leiomyomas.

\section{Acknowledgement}

This research was supported by Basic Science Research Program through the National Research Foundation of Korea (NRF) funded by the Ministry of Education (2017R1D1A1B03028045).

\section{Competing Interests}

The authors have declared that no competing interest exists.

\section{References}

1. Ryan GL, Syrop CH, Van Voorhis BJ. Role, epidemiology, and natural history of benign uterine mass lesions. Clin Obstet Gynecol. 2005; 48: 312-24.

2. Wallach EE, Vlahos NF. Uterine myomas: an overview of development, clinical features, and management. Obstet Gynecol. 2004; 104: 393-406.

3. Wilcox LS, Koonin LM, Pokras R, Strauss LT, Xia Z, Peterson HB. Hysterectomy in the United States, 1988-1990. Obstet Gynecol. 1994; 83: 549-55.

4. [http://www.sirweb.org/patients/uterine-fibroids/].

5. Scheurig-Muenkler C, Koesters C, Powerski MJ, Grieser C, Froeling V, Kroencke TJ. Clinical long-term outcome after uterine artery embolization: sustained symptom control and improvement of quality of life. J Vasc Interv Radiol. 2013; 24: 765-71.

6. Spies JB, Myers ER, Worthington-Kirsch R, Mulgund J, Goodwin S, Mauro M. The FIBROID Registry: symptom and quality-of-life status 1 year after therapy. Obstet Gynecol. 2005; 106: 1309-18.

7. Spies JB, Roth AR, Jha RC, et al. Leiomyomata treated with uterine artery embolization: factors associated with successful symptom and imaging outcome. Radiology. 2002; 222: 45-52.

8. Kroncke TJ, Gauruder-Burmester A, Scheurig C, et al. [Transarterial embolization for uterine fibroids: clinical success rate and results of magnetic resonance imaging]. Rofo. 2005; 177: 89-98.

9. Schwartz LB, Panageas E, Lange R, Rizzo J, Comite F, McCarthy S. Female pelvis: impact of MR imaging on treatment decisions and net cost analysis. Radiology. 1994; 192: 55-60.

10. Jha RC, Ascher SM, Imaoka I, Spies JB. Symptomatic fibroleiomyomata: MR imaging of the uterus before and after uterine arterial embolization. Radiology. 2000; 217: 228-35.

11. Katsumori T, Akazawa K, Mihara T. Uterine artery embolization for pedunculated subserosal fibroids. AJR Am J Roentgenol. 2005; 184: 399-402.

12. Scheurig-Muenkler $C_{1}$ Koesters $C_{2}$ Grieser C Hamm B, Kroencke TJ. Treatment failure after uterine artery embolization: prospective cohort study with multifactorial analysis of possible predictors of long-term outcome. Eur J Radiol. 2012; 81: e727-31.

13. Sipola P, Ruuskanen A, Yawu L, et al. Preinterventional quantitative magnetic resonance imaging predicts uterus and leiomyoma size reduction after uterine artery embolization. J Magn Reson Imaging. 2010; 31: 617-24.
14. Chang S, Kim MD, Lee M, et al. Uterine artery embolization for symptomatic fibroids with high signal intensity on T2-weighted MR imaging. Korean J Radiol. 2012; 13: 618-24.

15. Lee MS, Kim MD, Jung DC, et al. Apparent diffusion coefficient of uterine leiomyoma as a predictor of the potential response to uterine artery embolization. J Vasc Interv Radiol. 2013; 24: 1361-5.

16. Munro MG, Critchley HO, Broder MS, Fraser IS, Disorders FWGoM. FIGO classification system (PALM-COEIN) for causes of abnormal uterine bleeding in nongravid women of reproductive age. Int J Gynaecol Obstet. 2011; 113: 3-13.

17. Zlotnik E, de Lorenzo Messina M, Nasser F, et al. Predictive factors for pelvic magnetic resonance in response to arterial embolization of a uterine leiomyoma. Clinics (Sao Paulo). 2014; 69: 185-9.

18. Heinze G. A comparative investigation of methods for logistic regression with separated or nearly separated data. Stat Med. 2006; 25: 4216-26.

19. Gupta JK, Sinha A, Lumsden MA, Hickey M. Uterine artery embolization for symptomatic uterine fibroids. Cochrane Database Syst Rev. 2012; 5: Cd005073.

20. Naguib NN, Mbalisike E, Nour-Eldin NE, et al. Leiomyoma volume changes at follow-up after uterine artery embolization: correlation with the initial leiomyoma volume and location. J Vasc Interv Radiol. 2010; 21: 490-5.

21. Koesters C, Powerski MJ, Froeling V, Kroencke TJ, Scheurig-Muenkler C. Uterine artery embolization in single symptomatic leiomyoma: do anatomical imaging criteria predict clinical presentation and long-term outcome? Acta Radiol. 2014; 55: 441-9.

22. Firouznia K, Ghanaati H, Sanaati M, Jalali AH, Shakiba M. Uterine artery embolization in 101 cases of uterine fibroids: do size, location, and number of fibroids affect therapeutic success and complications? Cardiovasc Intervent Radiol. 2008; 31: 521-6.

23. Hecht EM, Do RK, Kang SK, Bennett GL, Babb JS, Clark TW. Diffusion-weighted imaging for prediction of volumetric response of leiomyomas following uterine artery embolization: a preliminary study. J Magn Reson Imaging. 2011; 33: 641-6.

24. Parthipun AA, Taylor J, Manyonda I, Belli AM. Does size really matter? Analysis of the effect of large fibroids and uterine volumes on complication rates of uterine artery embolisation. Cardiovasc Intervent Radiol. 2010; 33: 955-9.

25. Choi HJ, Jeon GS, Kim MD, Lee JT, Yoon JH. Is uterine artery embolization for patients with large myomas safe and effective? A retrospective comparative study in 323 patients. J Vasc Interv Radiol. 2013; 24: 772-8.

26. Isonishi S, Coleman RL, Hirama M, et al. Analysis of prognostic factors for patients with leiomyoma treated with uterine arterial embolization. Am J Obstet Gynecol. 2008; 198: 270.e1-6.

27. Kroencke TJ, Scheurig C, Poellinger A, Gronewold M, Hamm B. Uterine Artery Embolization for Leiomyomas: Percentage of Infarction Predicts Clinical Outcome1. Radiology. 2010; 255: 834-41.

28. Noda Y, Kanematsu M, Goshima S, et al. Prediction of early response to uterine artery embolization in fibroids: value of MR signal intensity ratio. Magn Reson Imaging. 2015; 33: 51-5.

29. Oguchi O, Mori A, Kobayashi Y, Horiuchi A, Nikaido T, Fujii S. Prediction of histopathologic features and proliferative activity of uterine leiomyoma by magnetic resonance imaging prior to $\mathrm{GnRH}$ analogue therapy: correlation between T2-weighted images and effect of GnRH analogue. J Obstet Gynaecol (Tokyo 1995). 1995; 21: 107-17.

30. Yamashita $Y$, Torashima $M$, Takahashi $M$, et al. Hyperintense uterine leiomyoma at T2-weighted MR imaging: differentiation with dynamic enhanced MR imaging and clinical implications. Radiology. 1993; 189: 721-5. 